\title{
Resistance of Oryza nivara and Oryza eichingeri derived lines to brown planthopper, Nilaparvata lugens (Stal)
}

\author{
S.A.P. Madurangi ${ }^{1}$, W.L.G. Samarasinghe ${ }^{2}$, S.G.J.N. Senanayake ${ }^{1^{*}}$, P.V. Hemachandra ${ }^{2}$ and Disna \\ Ratnasekera $^{1}$
}

${ }^{\prime}$ Department of Agricultural Biology, Faculty of Agriculture, University of Ruhuna, Mapalana, Kamburupitiya.

${ }^{2}$ Rice Research and Development Institute, Department of Agriculture, Ibbagamuwa, Bathalagoda.

Revised: 08 June 2010 ; Accepted: 21 January 2011

\begin{abstract}
This study evaluates the basis of brown planthopper (BPH) resistance in Oryza nivara (9864, WRAC 04), Oryza eichingeri (TC 01, TC 02), IR 54751-2-34-10-6-2 (an introgression line of Oryza officinalis), Ptb 33, Bg 380 and Bg $379 / 2$ accessions by screening for resistance, and characterizes the genes by molecular techniques. Four BPH populations reared on Ptb33, Bg 380, Bg 379/2, IR 54751-2-34-10-6-2 and a virulent BPH population collected from rice fields in Kegalle area Sri Lanka were used for screening tests. Screening for resistance was conducted following the standard honeydew test and molecular screening was carried out using specific molecular markers for $b p h 2, B p h 10$ and Bph13 genes. Results of the screening tests for resistance showed that the level of resistance of the tested wild rice accessions (O. nivara and $O$. eichingeri) was not significantly different from that of Ptb 33. These accessions also showed high level of resistance to all the BPH populations used in this study. Molecular screening revealed positive results with $O$. nivara (WRAC 04) accession for bph 2 and Bph 13 markers and $O$. eichingeri derived lines (TC $01 \&$ TC 02) for Bph 13 marker. Results showed that the emergence of virulent biotype in field rice cultivations have the potential to overcome the BPH resistance in $\mathrm{Bg} 379 / 2$.
\end{abstract}

Keywords: Brown planthopper, honeydew test, molecular screening, Oryza nivara, Oryza eichingeri, wild rice.

\section{INTRODUCTION}

The brown planthopper (BPH), Nilaparvata lugens (Stal) (Homoptera: Delphacidae) is one of the most serious rice pests in Sri Lanka. Annually BPH damage leads to a loss of about $5-10 \%$ of national rice production. At present, farmers depend mostly on chemical pesticides for the control of this pest (Kudagamage \& Nugaliyadda, 1995). This dependence leads to many adverse effects. The most effective way of BPH management is considered as host plant resistance. Varietal resistance is the most economic, least complicated and environmentally friendly approach for the control of insect pest damage (Pathak \& Kush, 1979). Rice is an annual grass that belongs to genus Oryza, which includes 22 wild species. These wild species of rice contain genes, which can be used for improvement of rice. Identification and characterization of these are important for their future transfer from wild rice to cultivated rice varieties. Researchers at the Rice Research and Development Institute (RRDI) at Bathalagoda use some BPH resistant lines developed from $O$. officinalis and $O$. australiensis in their breeding programmes. Fundamental studies have been undertaken to evalute the use of $O$. nivara and $O$. eichingeri in rice breeding programmes in Sri Lanka. BPH resistance has been detected in $O$. nivara and introgression lines derived from $O$. eichingeri and $O$. sativa at the RRDI (Kumari et al., 2007; Hemachandra et al., 2008). Therefore, understanding the basis of BPH resistance in these species will be very useful for varietal improvement programmes of rice in Sri Lanka. Bioassay of honeydew extraction has been effectively used for screening resistant lines (Heinrichs et al., 1985).

Molecular markers have become efficient tools for screening of BPH resistance and marker assisted selection (MAS) in rice. They have been helpful for the identification of donors of BPH resistance and the genetic study of BPH resistance (Su et al., 2006). Over 18 major BPH resistant genes have already been identified and characterized (Jena et al., 2006). Of these resistant genes Bph1, bph2, Bph9 and Bph10 were located on chromosome 12, Bph3 and bph12 were located on chromosome 4, bph4 was located on chromosome 6, 
Bph6 was located on chromosome11 and bph11 and Bph13 were located on chromosome 3 (Ishii et al., 1994; Hirabayashi et al., 1999; Renganayaki et al., 2002; Jena et al., 2003; Sharma et al. 2003). Primers specific to above resistant genes can be used to identify BPH resistant genes in $O$. nivara and $O$. eichingeri derived lines. The present study screened the above lines for BPH resistance using honeydew extraction bioassay and polymerase chain reaction (PCR) analysis using the sequence tagged site (STS) markers such as KAM4 (bph2), RG457 (Bph10) and AJ 096 (Bph13).

\section{METHODS AND MATERIALS}

O. nivara wild accessions (9864, WRAC 04), introgression lines of $O$. eichingeri and $O$. sativa (Pachchaperumal) (TC 01, TC 02), IR 54751-2-34-10-6-2 (introgression line of $O$. officinalis, a moderately resistant check) and Ptb 33 (resistant check), Bg 380 (susceptible check), Bg 379/2(moderately resistant check) varieties were used for both molecular and BPH bioassay studies (Khush, 1979; Renganayake et al., 2002).

Four BPH populations developed from BPH culture maintained at the RRDI, Batalagoda and virulent $\mathrm{BPH}$ population collected from fields in Kegalle and reared on $\mathrm{Bg} \mathrm{379/2}$ at Bathalagoda in separate cages (volume; $1.215 \mathrm{~m}^{3}$ ) were cultured as described in Tables $1 \& 2$ in a plant house at the RRDI, Bathalagoda for 3 months were used in this study.

The rice lines and varieties (9864, WRAC 04, TC 01, TC 02, IR 54751-2-34-10-6-2, Ptb 33, Bg 379/2 and $\mathrm{Bg} 380$ ) were grown singly in $15 \mathrm{~cm}$ clay pots and managed free from BPH infestations. Screening for resistance was done when they reached 2 months in age. Screening was done inside feeding chambers. Whatman No.2 filter paper stained with bromocresol green pigment
( $2 \mathrm{mg} / 1 \mathrm{~mL}$ of $70 \%$ ethanol) was placed around the base of the test plant before infesting $\mathrm{BPH}$ for screening. A pair of BPH females previously starved for 4 to $5 \mathrm{~h}$ were placed in the feeding chamber and allowed to feed for $24 \mathrm{~h}$. Honeydew excreted by BPH was absorbed to filter paper, which appeared as a blue spot. The area of the spot was assumed to be proportional to the honeydew production and amount of BPH feeding (Heinrichs et al., 1985). Reaction of BPH to host plants and plant resistance to $\mathrm{BPH}$ were determined based on honeydew production. Screening of rice lines and varieties for resistance was done following the randomized complete block design with five replicates.

Table 1: Description of BPH populations used for BPH Bioassay

\begin{tabular}{cl}
\hline Population no & \multicolumn{1}{c}{ Description } \\
\hline 1 & $\begin{array}{l}\text { BPH culture maintained at RRDI, Bathalagoda } \\
\text { on susceptible rice variety, Bg } 380 \text { for years and } \\
\text { subsequently continued culturing on Ptb } 33 \text { for } \\
3 \text { months }\end{array}$ \\
& $\begin{array}{l}\text { BPH culture maintained at RRDI on susceptible } \\
\text { rice variety, Bg } 380 \text { for years and subsequently } \\
\text { continued culturing on Bg 379-2 for 3 months }\end{array}$ \\
& $\begin{array}{l}\text { BPH culture maintained at RRDI on susceptible } \\
\text { rice variety, Bg } 380 \text { for years and subsequently } \\
\text { continued culturing on IR 54751-2-34-10-6-2 } \\
\text { for } 3 \text { months }\end{array}$ \\
& $\begin{array}{l}\text { Virulent BPH population collected from rice } \\
\text { cultivated fields of Bg 379/2 in Kegalle and } \\
\text { maintained at RRDI on rice variety, Bg 379/2 }\end{array}$ \\
& $\begin{array}{l}\text { BPH culture maintained at RRDI on susceptible } \\
\text { rice variety, Bg 380 }\end{array}$ \\
5 &
\end{tabular}

Table 2: Status of BPH resistance in host plants used in BPH cultures

\begin{tabular}{|c|c|c|}
\hline $\begin{array}{l}\text { Rice variety / line and status } \\
\text { of resistance }\end{array}$ & $\begin{array}{l}\text { Designated resistant } \\
\text { genes / source } \\
\text { of resistance }\end{array}$ & Reference \\
\hline 1. Bg $380-$ susceptible variety & ------- & (Khush, 1979) \\
\hline 2. Ptb $33 \quad-$ resistant variety & bph 2 and Bph 3 & (Khush, 1979) \\
\hline $\begin{aligned} & \text { 3. Bg } 379-2- \text { moderately } \\
& \text { resistant variety }\end{aligned}$ & Bph 3 - Ptb 33 & (Khush, 1979) \\
\hline $\begin{aligned} \text { 4. IR54751-2-34-10-6-2 - moderately } & \text { resistant line }\end{aligned}$ & $\begin{array}{l}\text { bph } 11 \text {, bph } 12 \text { and Bph } \\
13-\text { Oryza officinalis }\end{array}$ & (Renganayaki et al., 2002) \\
\hline
\end{tabular}


DNA was extracted following the CTAB "Miniprep" protocol, (Anonymous, 2003). DNA extracted from the rice lines and varieties were screened by PCR amplification using RG 457 FL/RL (Bph 10), KAM 4 (bph 2) and AJ096 (F/R) (Bph 13) STS primers.

The PCR mixture contained 20 - $100 \mathrm{ng}$ template DNA, 1 pmol of each primers, $0.25 \mathrm{mM}$ dNTP's each, $1 \mathrm{X}$ buffer (20 mM Tris $\mathrm{pH} 8.0$ including $20 \mathrm{mM} \mathrm{MgCl}_{2}$ ) and 0.5 units of Taq polymerase in a total volume of 10 - $15 \mu \mathrm{L}$. PCR was performed by using a Master Cycler Gradient. PCR mixtures were initially denatured at $94{ }^{\circ} \mathrm{C}$ at $5 \mathrm{~min}$. This was followed by 40 cycles of PCR amplification using following parameters: denaturation of $94{ }^{\circ} \mathrm{C}(30 \mathrm{~s})$, primer annealing at $66{ }^{\circ} \mathrm{C}(1 \mathrm{~min})$ and primer extension at $72{ }^{\circ} \mathrm{C}(1 \mathrm{~min})$. After PCR, products of $\mathrm{RG} 457$ primer were subjected to digestion by restriction enzyme Hinfl incubating at $37^{\circ} \mathrm{C}$ for $1.5 \mathrm{~h}$. PCR products were electrophorated in 1.4\% agarose gel to separate the amplified DNA fragments. The gel was subsequently stained by ethidium bromide and amplified DNA fragments were visualized under UV light as bands. PCR products of AJ096 primer were electrophorated in $10 \%$ Silver stained urea PAGE to confirm the separated bands in $1.4 \%$ agarose gel.



\section{RESULTS}

PCR amplifications with KAM 4 primer gave rise products of 300bp, (Murai et al., 2001) with Ptb 33 and WRAC 04 (Figure 1-A). This indicates the presence of $b p h 2$ gene in the genome of Ptb 33 and WRAC 04 accessions. Gene bph 2 is one of the $12 \mathrm{BPH}$ resistant genes so far identified in several indica cultivars. Ptb 33 shows high level of BPH resistance. Khush (1979) reported that it was due to combination of two different resistant genes, bph 2 and $B p h 3$. PCR analysis with $\mathrm{Bg}$ $379 / 2$, the variety developed by RRDI, Bathalagoda using Ptb 33 as the source of resistance, did not indicate bph2 gene in its genome (Khush, 1979).

PCR amplifications with AJ 096 primer gave rise to a $200 \mathrm{bp}$ DNA fragment indicating the presence of resistant gene Bph13 and a $179 \mathrm{bp}$ fragment indicating the presence of a susceptible gene in the genome (Renganayaki et al., 2002). PCR analysis indicated the presence of Bph13 gene in $\mathrm{Bg} 357, \mathrm{Bg} 379 / 2, \mathrm{Bg} 380$, IR 54751-2-34-106-2 (IR line), O. nivara (WRAC 04), O. eichingeri (TC 01 and TC 02) accessions and but not in Ptb 33 and O. nivara (9864) accession (Figure 1-B).

Figure 1: PCR amplification of DNA of tested rice entries by KAM 4 primer followed by electrophoration in $1.4 \%$ agarose gel (A), by AJ096 primer followed by electrophoration in $1.4 \%$ agarose gel (B), by RG $457 \mathrm{FL} / \mathrm{RL}$ primers followed by electrophoration in $1.4 \%$ agarose gel (C) and digested products of (C) by restriction enzyme Hinfl followed by electrophoration in $1.4 \%$ agarose gel (D) 
A

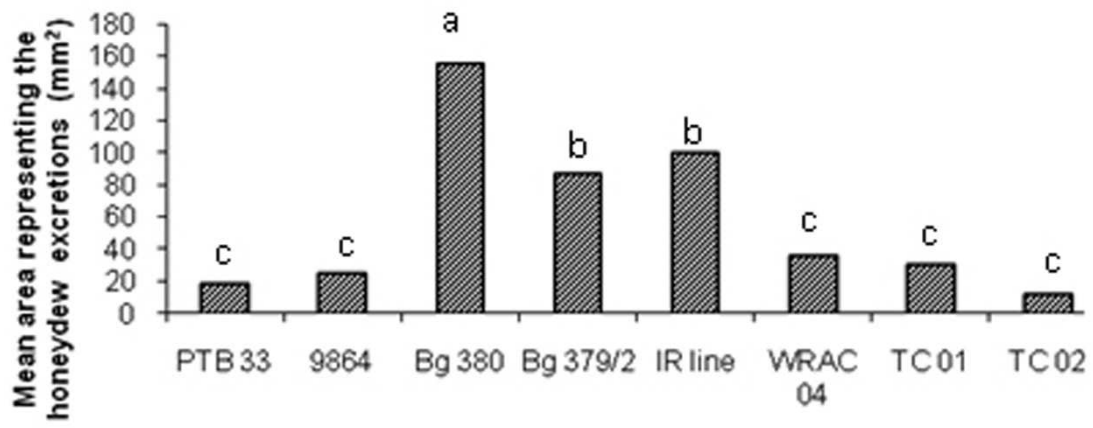

Variety

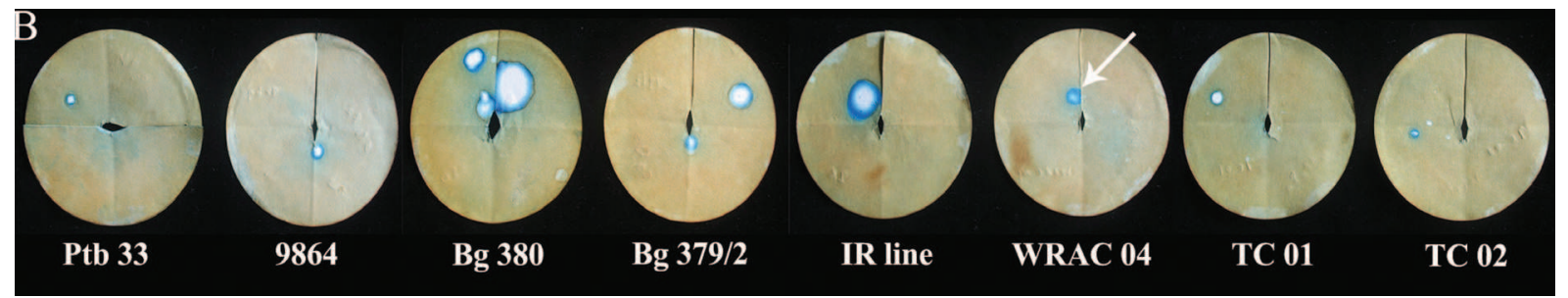

Figure 2: Honeydew productions in tested rice lines and varieties

A. Mean area representing honeydew excretion $\left(\mathrm{mm}^{2}\right)$ in tested rice lines and varieties

B. Honeydew excretions in tested rice lines and varieties visualized by using bromocresol green treated filter papers (white arrow shows honeydew spot)

Means with the same letter are not significantly different at $5 \%$ level (DMRT).

PCR analysis with forward and reverse primers of RG 457 showed amplification of 750bp DNA fragment with DNA of all the tested lines and varieties (Figure 1-C) (Nguyen et al., 1999). These amplified products were detected only after digestion by Hinfl restriction enzyme. Further analysis of them showed DNA fragments of $300 \mathrm{bp}, 250 \mathrm{bp}$ and $200 \mathrm{bp}$ in the amplified products of Bg 357 and DNA fragments of 550bp and 200bp in the amplified products of all the other entries (Figure 1-D). This indicated the presence of Bphl0 gene only in the genome of Bg 357(Nguyen et al., 1999).

Analysis of honeydew productions showed a significant difference among the tested rice lines and varieties $(\alpha=0.05)$ (Figure 2-A). O. nivara (WRAC 04, 9864), O. eichingeri (TC 01, TC 02) and Ptb 33 showed significantly lower honeydew productions. This indicated their resistance to BPH. Bg 380 showed significantly higher honeydew production indicating no resistance to BPH. Moderate but significantly different honeydew production was observed in $\mathrm{Bg} 379 / 2$ and IR line (Figure 2-B) indicating moderate resistance to BPH $(\mathrm{CV}=42 \%)$.
Figure $3 \mathrm{~A}$ shows the mean areas of honeydew excretions in relation to tested BPH populations on all the tested lines and varieties. Analysis of mean areas of honeydew excretions showed a significant difference among populations $(\alpha=0.05)$. The significantly high honeydew excretions were produced by populations reared on $\mathrm{Bg} 379 / 2$ and the virulent population of Kegalle. The significantly low honeydew excretions were produced by populations reared on IR 54751-2-34-10-6-2 and Bg 380. The population reared on Ptb 33 showed no significant difference of honeydew excretions to any populations. Thus, virulence of the Kegalle population and the population reared on Bg 379/2 were significantly higher than that of populations reared on IR 54751-234-10-6-2 and $\mathrm{Bg} 380$. This also supports the report of the emergence of new biotype in cultivated fields of $\mathrm{Bg}$ 379/2 variety in Kegalle (Fernando et al., 2007).

Data of honeydew excretions also indicated a significant interaction between different populations and tested varieties $(\alpha=0.05)$ (Figure 3-B). Bg 380 variety was highly susceptible to BPH populations reared on Ptb 33 and $\mathrm{Bg} 379 / 2$ and the virulent population of Kegalle. 

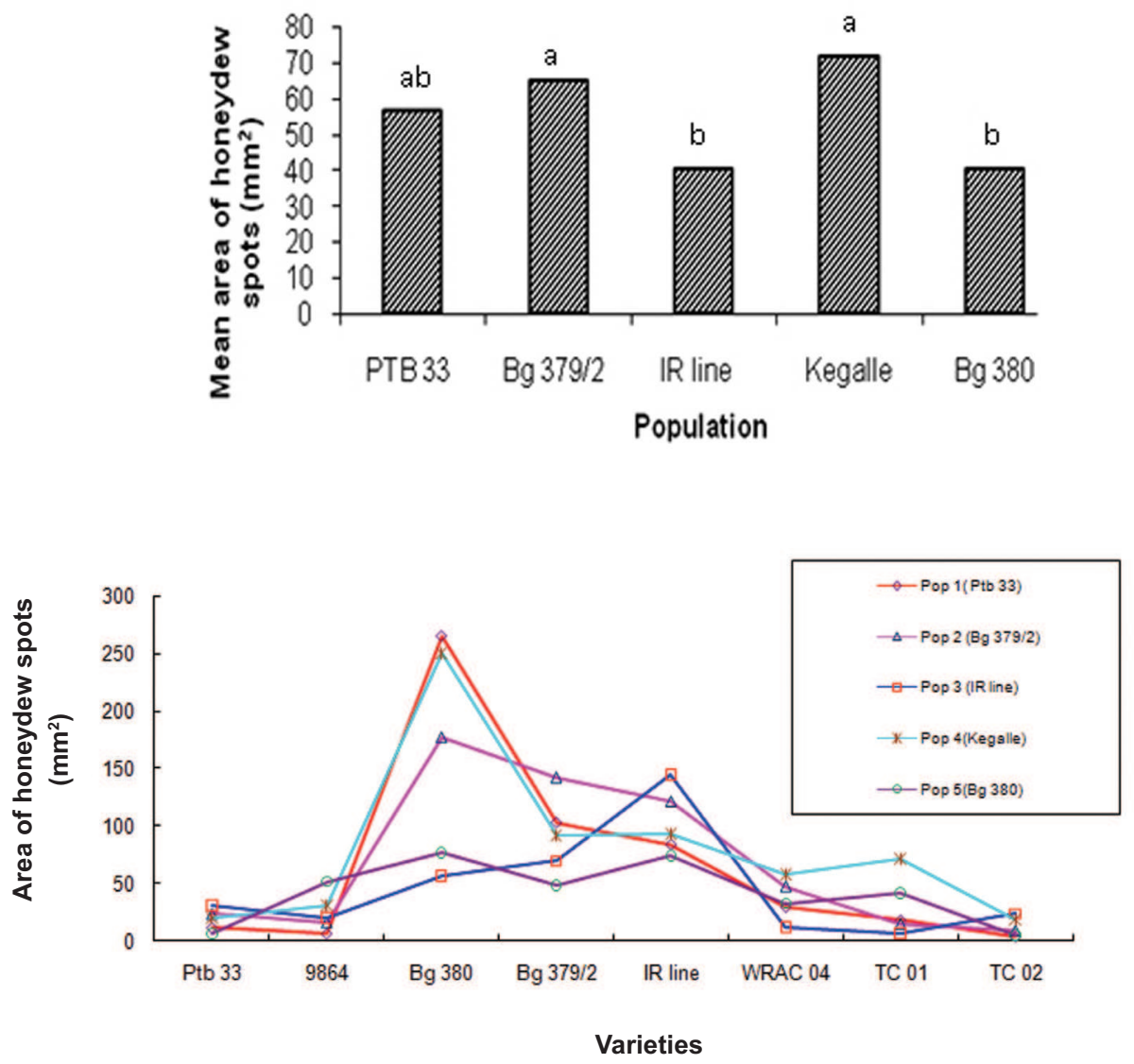

Figure 3: Mean area $\left(\mathrm{mm}^{2}\right)$ representing honeydew excretion by $5 \mathrm{BPH}$ populations when fed on tested rice lines and varieties (A) and interaction between $\mathrm{BPH}$ populations and tested rice lines and varieties (B); Means with the same letter are not significantly different at $5 \%$ level (DMRT).

However the IR line was most susceptible to the BPH population reared on IR line. The population reared on $\mathrm{Bg} 380$ caused more or less equal damage to $\mathrm{Bg} 380$ and IR 54751-2-34-10-6-2. However Ptb 33 and lines of $O$. nivara (WRAC 04, 9864) and $O$. eichingeri (TC 01, TC 02) showed almost complete resistance to all the $\mathrm{BPH}$ populations.

\section{DISCUSSION AND CONCLUSION}

$\mathrm{BPH}$ resistance has been identified in a wide range of traditional rice varieties and wild rice species (Pathak \& Khush, 1979; Heinrichs et al. 1985). Scientists have already characterized and exploited some of the genes responsible for resistance and upgraded cultivated rice varieties. To date these varieties play a significant role in controlling BPH outbreaks in rice cultivations. However development of virulent BPH populations is becoming a serious threat for rice cultivation at present. Thus, constant search for resistant genes is very important. Wild relatives of rice, $O$. australiensis and $O$. officinalis have already become donors of BPH resistance (Renganayaki et al., 2002). Several reports have highlighted wild rice species of O. latifolia, O. nivara, O. punctata and O. minuta as important sources of BPH resistance (Wu et al., 1986). Further research in other wild relatives is suggested as they may be useful as donors of BPH resistance in variety improvement programmes in the future.

Screening with specific molecular markers in this study was revealed the presence or absence of three BPH resistant genes (bph2, Bph10 and Bph13) in O. nivara and $O$. eichingeri derived lines. It has identified the presence of Bph10 gene in O. australiensis (Nguyen et al., 1999). This gene is absent in $O$. nivara and $O$. eichinigeri derived lines. The analysis showed the presence of $b p h 2$ gene in the genome of $O$. nivara (WRAC 04) accession. However, analysis did not indicate any evidence for the presence of bph 2 gene in O. nivara (9864) accession. Molecular analysis with markers indicated the presence 
of Bph 13 gene in $\mathrm{Bg} 380, \mathrm{Bg} 357, \mathrm{Bg} 379 / 2$, IR 54751-234-10-6-2 line, O. nivara (WRAC 04) and O. eichingeri derived lines (TC 01 and TC 02). Honeydew test results showed that $\mathrm{Bg} 380$ is susceptible to the $\mathrm{BPH}, \mathrm{Bg} 379 / 2$ and IR 54751-2-34-10-6-2 line moderately resistant to the BPH while, O.nivara (WRAC 04) and O.eichingeri (TC 01 and TC 02) resistant to the $\mathrm{BPH}$ and $\mathrm{Bg} 357$ known to be moderately resistant to the BPH. Although $\mathrm{Bg} 380$ is susceptible to the BPH it showed positive results for Bph 13 gene indicating Sri Lankan BPH is virulent to the $B p h 13$ gene. Hence resistance observed in Bg 379/2, IR 54751-2-34-10-6-2 line, O. nivara (WRAC 04), O. eichingeri (TC 01 and TC 02) and Bg 357 due to the presence of other resistant genes but not due to the presence of Bph 13 gene. The lower level of resistance in Bg 379/2 in comparison to Ptb 33 may be related to the absence of bph2 gene in BG 379/2, which is present in Ptb33.

Results of the honeydew test indicated resistance in Ptb 33 and all the tested wild rice accessions to all BPH populations. However their degree of resistance was not significantly different among each other. Therefore, the BPH resistance identified in O. nivara accession (9864), $O$. nivara accession (WRAC 04) and $O$. eichingeri derived lines (TC 01, TC 02) may be due to the presence of other resistant genes or new genes. Identification of additional BPH resistant genes and exploiting them to widen the genetic base of cultivated rice varieties need to be continued in view of overcoming future $\mathrm{BPH}$ outbreaks by emergence of new biotypes. Results of this study highlight the possibility of using genetic resources of wild rice, O. nivara and $O$. eichingeri in order to develop broad based and durable resistance for BPH in cultivated rice varieties in the future.

\section{Acknowledgement}

Authors acknowledge all members of the Entomology Division of the Rice Research and Development Institute, Bathalagoda, Ibbagamuwa and staff of the Biotechnology Division of the Plant Genetrc Resources Centre, Gannoruwa, Peradeniya, especially Mrs. H.M.P.S. Kumari as well as all the academic and non academic staff of the Department of Agricultural Biology, Faculty of Agriculture, University of Ruhuna, Mapalana, Kamburupitiya for their contribution to this study.

\section{REFERENCES}

1. Anonymous (2003). Workshop Handbook on Genomics and Crop Improvement, pp. 51-57. International Center for Genetic Engineering and Biotechnology, New Delhi,
India.

2. Fernando K.K.S., EkanayakeE.M.D.S.B.\& Ketipearachchi Y. (2007). Identification of biotypes of brown planthopper using microsatellite markers. Annals of Sri Lanka Department of Agriculture 9(1): 27-33.

3. Heinrichs E.A., Medrano F.G. \& Rapusas H.R. (1985). Genetic Evaluation for Insect Resistance in Rice, pp. 45173. International Rice Research Institute, Los Banos, Laguna, Philippines.

4. Hemachandra, P.V., Nawarathne, N.M.A., Dissanayake, D.W.A.J. \& Geethika, W.M.U.S. (2008). Wide hybridization studies between O.sativa (cultivated Asian type) and O. nivara (wild rice). Annals of Sri Lanka Department of Agriculture 10(1): 343.

5. Hirabayashi H., Kaji R., Angeles E.R., Ogawa T., Brar D.S. \& Khush G.S. (1999). RFLP analysis of a new gene for resistance to brown planthopper derived from O. officinalis on rice chromosome 4. Breed Science 1(1): 48.

6. Ishii T., Brar D.S., Multani D.S. \& Khush G.S. (1994). Molecular tagging of genes for brown planthopper resistance and earliness introgressed from Oryza australiensis into cultivated rice, O. sativa. Genome 37(2): 217-221.

7. Jena K.K., Jeung J.U., Lee J.H., Choi H.C. \& Brar D.S. (2006). High-resolution mapping of a new brown planthopper (BPH) resistance gene, Bph 18(t), and markerassisted selection for BPH resistance in rice (Oryza sativa L.). Theoretical and Applied Genetics 112(2): 288-297.

8. Jena K.K., Pasalu I.C., Rao Y.K., Varalaxmi Y., Krishnaiah K., Khush G.S. \& Kochert G. (2003). Molecular tagging of a gene for resistance to brown planthopper in rice (Oryza sativa L.). Euphytica 129(1): 81-88.

9. Khush G.S. (1979). Genetics of and breeding for resistance to the brown planthopper. In: Brown planthopper: Threat to rice production in Asia. Proceedings of the International Conference on Brown Planthopper, Los Banos, Laguna, Philippines, 16-19 November. International Rice Research Institute, Los Banos, Laguna, Philippines. pp. 321 - 332.

10. Kudagamage C. \& Nugaliyadde L. (1995). Present status and future direction of insect pest management in rice. In: Proceedings of the Rice Congress 1990 (eds. S.L.Amarasiri, K. Nagaraj \& B.M.K. Perera), Kandy, 3-4 September. Department of Agriculture, Peradeniya. pp. 39-50.

11. Kumari, H.M.P.S., Yatawara, I.W.M.K.N.K., Silva, U.N.D., Samarakoon, S.M.M., Samarakoon, Y.M.S.K., Abeysiriwardhna, D.S.Z. \& Rajapaksha, T. (2007). Introgression of economically important genes from wild rice Oryza eichingeri to cultivated rice Oryza sativa. Annals of Sri Lanka Department of Agriculture 9(1): 81-87.

12. Murai H., Hashimoto Z. \& Sharma P.N. (2001). Construction of a high resolution linkage map of rice brown planthopper (Nilaparvata lugens Stal) resistance gene bph-2. Theoretical and Applied Genetics 103(4): 526-532.

13. Nguyen T.L., Brar D., Khush G.S., Huang N. \& Buu B.C. (1999). Development of STS markers to identify brown planthopper resistance in a segregating population. Omonrice 7(1): 26-34. 
14. Pathak M.D. \& Khush G.S. (1979). Studies of varietal resistance in rice to brown planthopper at the International Rice Research Institute. In: Brown Planthopper: Threat to Rice Production in Asia. Proceedings of the International Conference on Brown Planthopper, Los Banos, Laguna, Philippines, 16-19 November. International Rice Research Institute, Los Banos, Laguna, Philippines, pp. 285-301.

15. Renganayaki K., Allan K.F. \& Sadasvam S. (2002). Mapping and progress toward map-based cloning of brown planthopper biotype-4 resistance gene introgressed from Oryza officinalis in to cultivated rice, O.sativa. Crop Science 42(6): 2112-2117.
16. Sharma P.N., Ketipearachchi Y., Murata K., Torii A., Takumi S., Mori N. \& Nakamura C. (2003). RFLP/AFLP mapping of a brown planthopper (Nilaparvata lugens Stal) resistance gene Bphl in rice. Euphytica 129(1): 109-117.

17. Su C.C., Zahi H.Q., Wang C.M., Sun L.H. \& Wan J.M. (2006). SSR mapping of brown planthopper resistant gene Bph9 in Kaharanama, an indica rice (Oryza sativa L.). Acata Genetica Sinica 33(3): 262-268.

18. Wu J.T., Heinrichs E.A. \& Medrana F.G. (1986). Resistance of wild rice Oryza spp. to the brown planthopper, Nilaparvata lugens (Homoptera: Delphacidae). Environmental Entomology 15(3): 648-653. 